\title{
Investigación
}

\section{El futuro de \\ la educación}

DOI: 10.29236/sistemas.n158a4

Las dinámicas del mundo complejo que enfrentamos necesitan de la educación como pieza clave para el desarrollo de las personas, sociedades del presente $y$ del futuro.

\section{Andrés R. Almanza J.}

\section{Resumen}

El presente documento recoge las principales consideraciones de algunos actores globales sobre cómo debe ser la educación del futuro. El objetivo es mostrar tendencias a nivel mundial, luego del fenómeno que vive la humanidad.

\section{Introducción}

Las complejas dinámicas a raíz de la pandemia producida por el $\mathrm{CO}$ VID-19, han transformado los aspectos de la vida y los diferentes sectores. Uno de ellos la educación con un abrupto cambio en la experiencia del aprendizaje y el acoplamiento a estas nuevas realidades. En las sociedades latinoamericanas pasar de lo presencial a lo virtual era algo inevitable, hecho que se presentó mucho antes de lo previsto (Castaño. F. 2020).
En esa dirección, la incertidumbre ha tomado fuerza y modelará no sólo el año 2021, sino los venideros, tal y como lo indica un reciente reporte del Centro de Pensamiento Global (CIDOB), (Soler, 2020).

En la reciente reunión del Foro Económico Mundial (WEF), celebrada en Davos, Suiza, la educación fue considerada como una fuente indispensable para un futuro sostenible que estará marcado por grandes cambios y que demanda re- 
pensar la experiencia de los aprendizajes en ambientes híbridos (Fore, $F, 2021$ ).

En consecuencia, este documento pretende revisar algunas de las visiones de los entes multilaterales como el Foro Económico Mundial, la Organización para la Cooperación y el Desarrollo Económicos (OECD), la Organización de las Naciones Unidas para la Educación, la Ciencia y la Cultura (Unesco) y otros documentos de referencia sobre el tema que nos ocupa, la educación del futuro.

\section{Educación del futuro y el futuro de la educación}

Existen muchos consensos a nivel global, regional y local sobre el impacto que la educación tiene para el cierre de las desigualdades en el mundo (Anderson. C., 2014). Distintos entes multilaterales en los procesos de la crisis del Covid-19 han resaltado la importancia de la educación, su futuro, sus impactos y las transformaciones que ha sufrido para ser un sector que se acomode a las realidades de los próximos años, precisamente por los importantes efectos relacionarlo con muchos aspectos de la vida humana, como el desarrollo personal, las sociedades, la economía e inclusive la política (Guterres. A., 2020).

De igual manera, la Agenda global de 2030 centra su atención en la educación como un pilar fundamental, así como una herramienta de progreso y sostenibilidad de las sociedades del presente y del futuro y es definida como un Objetivo de Desarrollo Sostenible (ODS), entre los 17 (ODS) de la agenda, aprobada por las Naciones Unidas. En uno de sus objetivos, específicamente el cuarto, indica: "Garantizar una educación de calidad inclusiva y equitativa y promover oportunidades de aprendizaje permanente para todos". Se trata de un motor fundamental para aspirar a que la sociedad logre adaptarse al mundo disruptivo que empieza a mostrar la nueva década (Martínez. P., Vialta. J., 2021).

Acontinuación, se relacionan las visiones más relevantes de algunos de los entes multilaterales que se han ocupado de considerar y reflexionar al respecto.

\section{Naciones Unidas (UN)}

En el informe titulado "La educación durante la Covid-19 (UN, 2020) y después de ella", las Naciones Unidas (UN) resaltan:

1. El sistema de educación a nivel global ha sufrido la mayor interrupción en la historia de los sistemas educativos; ha afectado a más de mil 600 millones de estudiantes, en más de 190 países. El $94 \%$ de los estudiantes en cualquier centro de enseñanza se ha visto afectado y asciende al $99 \%$ en los países de bajo y medio ingreso (Figura 1).

2. Por tal razón, la desigualdad crece y el acceso, oportunidad y posibilidad, tanto del aprendizaje 


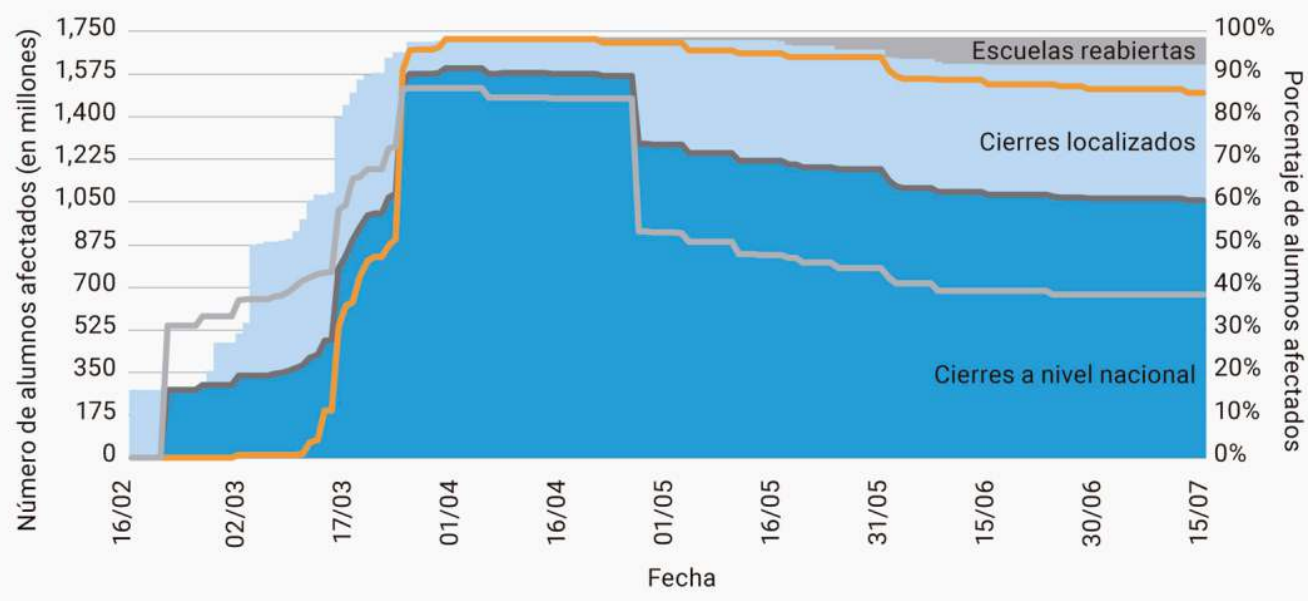

$\begin{array}{lcc}\text { - Porcentaje total de alumnos } & \text { Países de ingreso bajo } & \text { Países de ingreso } \\ \text { afectados por cierres a nivel nacional } & \text { y mediano bajo } & \text { mediano alto y alto }\end{array}$

Figura 1. Número de niños afectados por cierres de escuelas en el mundo

como de la enseñanza se han incrementado y así continuarán pasada la pandemia. Es probable que el abandono escolar pueda afectar alrededor de 23,8 millones de estudiantes alrededor del mundo, todo esto producto de los efectos sistémicos de la pandemia. Así como se registran los efectos de largo plazo en los procesos del aprendizaje de los jóvenes en todo el mundo, en esa misma medida el desarrollo humano que surge de la educación, también ha tenido fuertes impactos. Se estima que el índice de desarrollo humano disminuirá en un tercio y claramente tendrá efecto en la generación de estudiantes de todas las edades.

3. La financiación de la educación en todos los sectores también denota gran tensión y muestra que, para los países de ingresos bajos, y medios, se ha alcanzado una cifra cercana a los 148 millones de dólares (Figura 2).

4. Por otro lado, la innovación ha llegado a involucrarse para apoyar la continuidad de los sistemas educativos en todo el mundo. Se han aprendido nuevas cosas, la presencia de ambientes a distancia ha sido uno de los pilares identificados y, pasada la pandemia, los ambientes híbridos predominarán y la tecnología, la innovación tendrán roles preponderantes.

Así las cosas, la UN propone 5 estrategias fundamentales para repensar la educación del futuro construyéndola desde el presente:

1. Salud y educación juntas. La reapertura debe ser progresiva y repensada minuciosamente. 


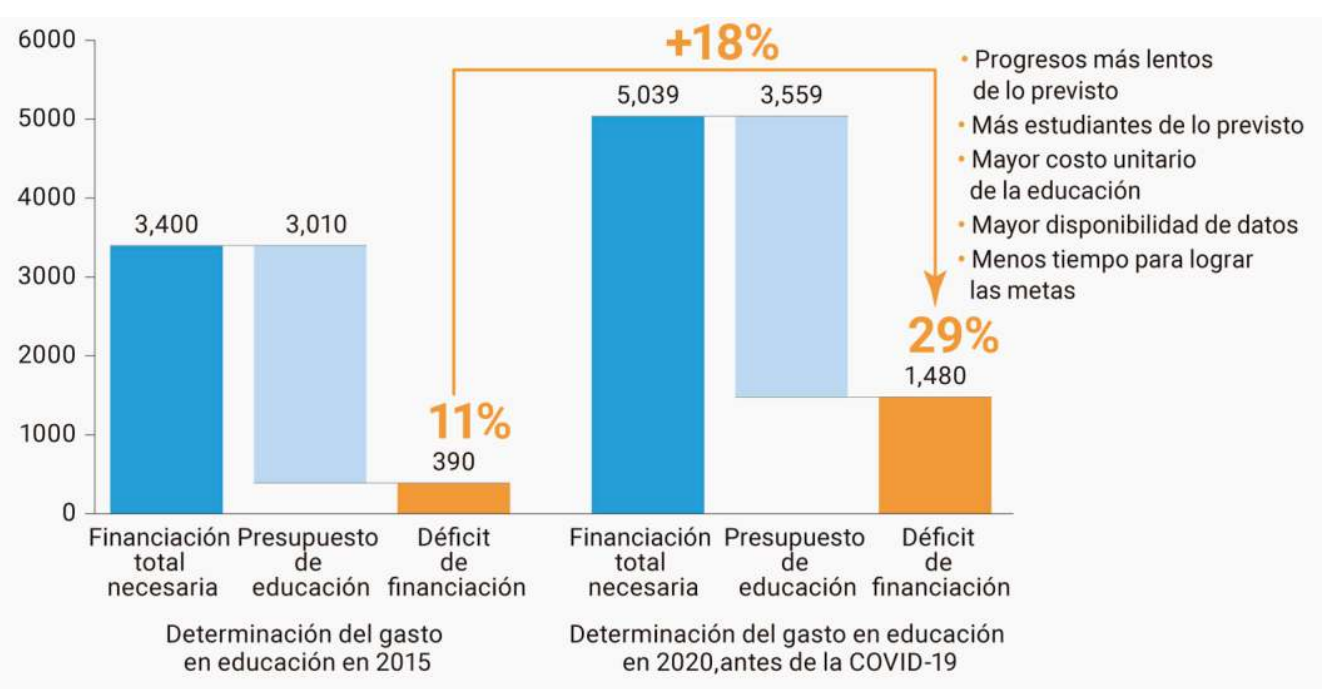

Fuente: UNESCO, Informe de seguimiento de la educación en el mundo (2020). New realities for education affected by COVID Cost predictions.

Figura 2. Déficit de financiación antes de la COVID-19 para lograr el objetivo de desarrollo sostenible 4 (en millones de dólares)

2. Proteger de manera inteligente la financiación de la educación, coordinar esfuerzos para que existan resultados.

3. Repensar la educación en torno al aprendizaje y al modelo de enseñanza.

4. Fortalecer la resiliencia del sistema educativo para que sea equilibrado, sostenible y equitativo.

\section{Foro Económico Mundial (FEM)}

Para (FEM) no es distante el tema y es un poco más amplio, en la medida en que hablar de educación es referirse a los efectos del desarrollo futuro.

En su reporte denominado "Escuelas del Futuro" (FEM,2020), publicado antes del proceso de la pandemia, considera que la educación y las escuelas del futuro deben pensar en una evolución que involucre calidad desde ocho dimensiones:

1. Ciudadanos que tengan un pensamiento global.

2. La creatividad e innovación como fuente del desarrollo de las personas.

3. Las personas con una aproximación a lo digital.

4. El mundo de las personas.

5. Los procesos de aprendizajes más dinámicos, personales e interactivos.

6. Aprendizaje inclusivo y accesible.

7. Resolución de problemas considerando la cooperación y colaboración. 
8. El aprendizaje como un proceso de vida, no como productos terminados (Figura 3).

De igual manera, en su reporte sobre el trabajo del futuro (FEMb, 2020) menciona que es necesario reforzar los procesos de educación de las personas, para que rápidamente se adapten a los nuevos puestos de trabajo que están surgiendo y para los cuales las brechas se van a incrementar. Además, sostiene que los empleos del futuro dependerán cada vez más de la educación de las personas y por tanto es necesario cerrar las brechas. Muestra (WEF) la necesidad de desarrollar enfoques sistémicos que permitan articular la coordinación entre la academia en todos sus niveles y el sector empresarial tanto público como privado, de tal forma que los esfuerzos por reentrenar y fortalecer las habilidades, así como las capacidades, co- bren importancia para cerrar las brechas y atender los desafíos futuros (Figura 4).

En ese mismo informe surge la pregunta de la relación que existe hoy entre las habilidades necesarias para desempeñarse en la vida laboral, y lo que sucede en todos los niveles de formación, pensando si los centros de formación, no solo las universidades, sino todos los niveles están pensando en las habilidades y capacidades requeridas para desempeñarse en la vida y en la sociedad (Bast. G., 2019).

En las reuniones pública del Foro Económico Mundial en Davos, fueron planteados elementos claves sobre la educación del futuro, en el marco de las siguientes consideraciones:

1. Es necesario que la educación del futuro considere las habili-
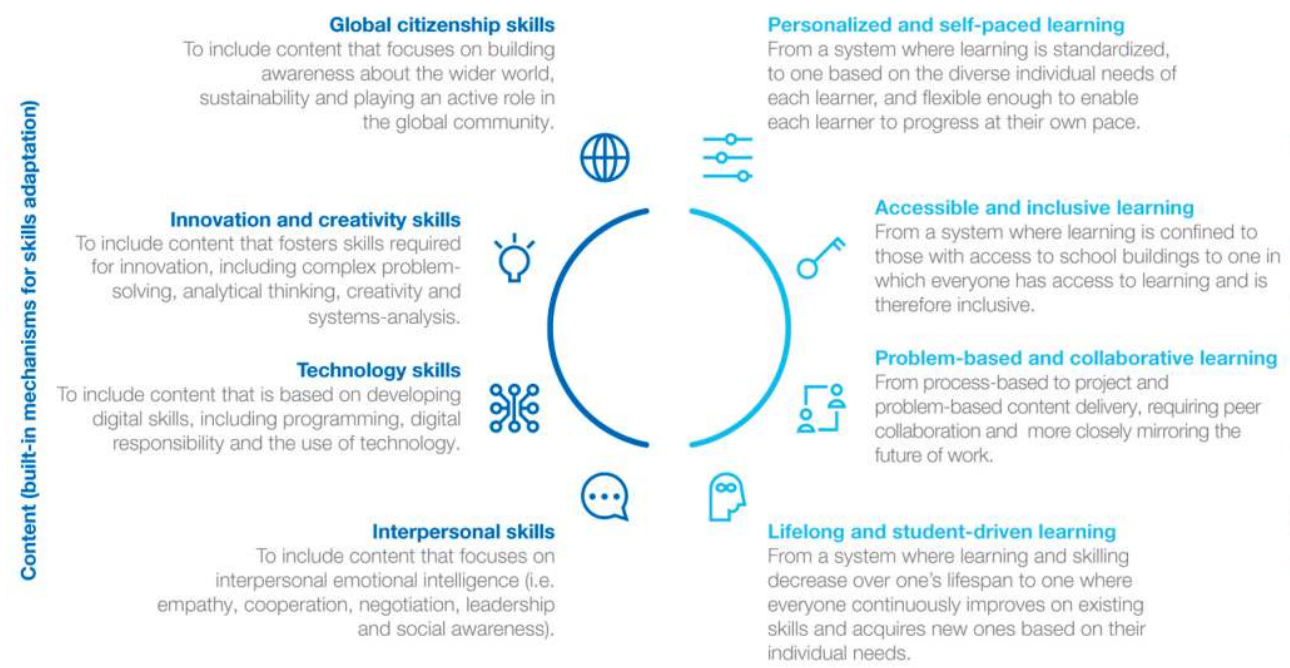

Figura 3. 


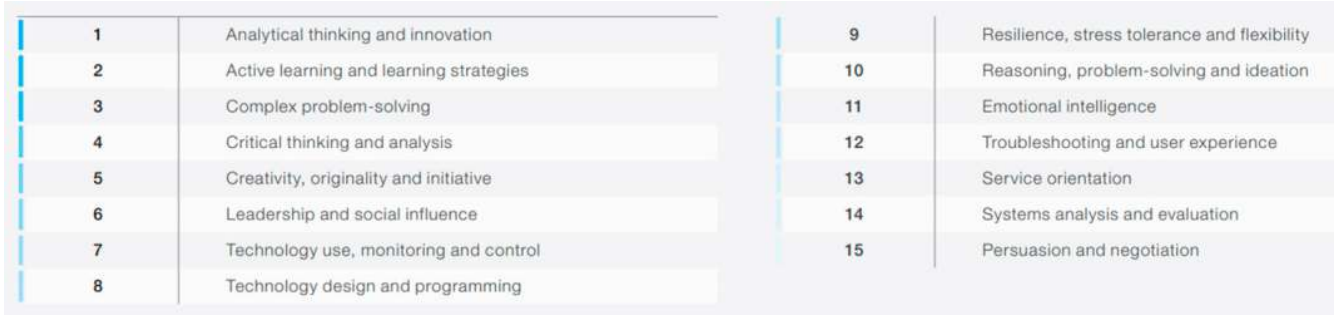

Figura 4.

dades esenciales, las digitales y las ocupacionales como fuente fundamental del desarrollo de las próximas generaciones.

2. La conectividad es esencial para la inclusión y cobertura.

3. La cooperación entre lo público y privado es esencial para el desarrollo de la educación en todos los niveles.

4. Los ambientes híbridos del futuro requieren de experiencias de aprendizajes diferentes, se requiere repensar los modelos existentes.

5. La educación de nivel superior debe pasar por un proceso de reinvención que considere lo global y lo digital en el proceso.

6. Se requiere crear comunidades de 'aprendedores' y aprendizajes que permitan acelerar el proceso.

7. Repensar los currículos restrictos que pueden ser obstáculos para las habilidades de innovación y creatividad (Fore. F. Gurg. A., Fortier. S. Goodwin. J., 2021).

\section{UNESCO}

Desde el año 2019 la UNESCO luego de la presentación de la Agenda 2030 de la OECD, lanzó su iniciativa denominada "los futuros de la educación, aprender a transformarse", enfocada en el horizonte de la educación hacia el año 2050, buscando la manera de reinventar aspectos conducentes a que la educación y el conocimiento sean un aporte para las economías a nivel global, (UNESCO, 2019) (Figura 5).

En términos de datos reales plasmados por la UNESCO sobre la educación y lo que ha sucedido frente a la pandemia, se menciona que: más de 800 millones de habitantes, un año después de la pandemia, permanecen afectados por los cierres de las universidades, más de la mitad de la población mundial (UNESCO, 2021).

La figura 6 que corresponde a las mediciones propias de la UNES$\mathrm{CO}$ en relación con el cierre de las aulas en todos los niveles muestra que la media de cierre completo de las escuelas fue de 3,5 meses, elevado en ciertas poblaciones a 5,5 meses, lo que equivale a dos tercios de un año académico.

Considerando ese panorama toma mayor fuerza su programa orientado a realizar transformaciones pro- 


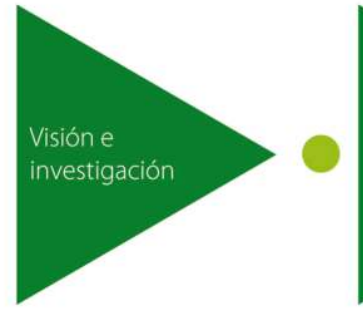

Octubre 2019 -

septiembre 2020

Debate sobre los desafios y las oportunidades de la educación previstos para 2050 y más allá

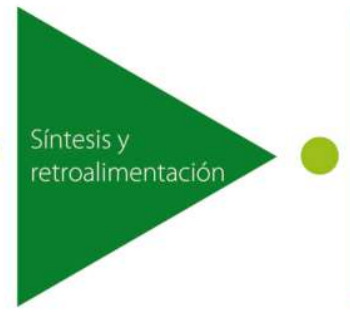

Octubre 2020 - marzo 2021

Deliberación sobre las

visiones y estrategias iniciales propuestas por la comisión internacional

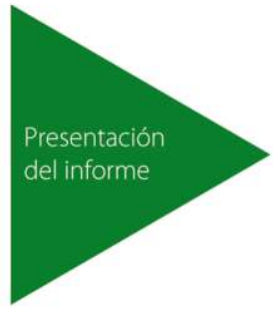

Noviembre 2021

Publicación del informe en la

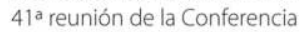
General de la UNESCO

Figura 5. Cronograma y Fases

fundas alrededor de componentes fundamentales tales como:

1. Calidad y pluralidad de las realidades del mundo, para tener acceso a ellas y que la calidad sea homogénea.

2. Enfoque de desarrollo humano de la educación, en el que la sostenibilidad de todo el modelo sea el bien común a alcanzar.

3. El conocimiento como bien común y de soporte, aunados a los criterios anteriores para promover el desarrollo.
4. Marcos del aprendizaje, en la búsqueda de modelos de enseñanza, el soporte de lo nuevo y los enfoques en las prácticas del aprender.

5. Democratización y acceso a la educación por parte de todas las personas.

6. Equidad e inclusión como herramientas que permitan una cobertura amplia y sólida.

Aspectos dirigidos hacia: a) Sostenibilidad de la humanidad y del planeta, b) Producción de conoci-
?

1-10 weeks

- 11-20 weeks

21-30 weeks

- 31-40 weeks

$41+$ weeks

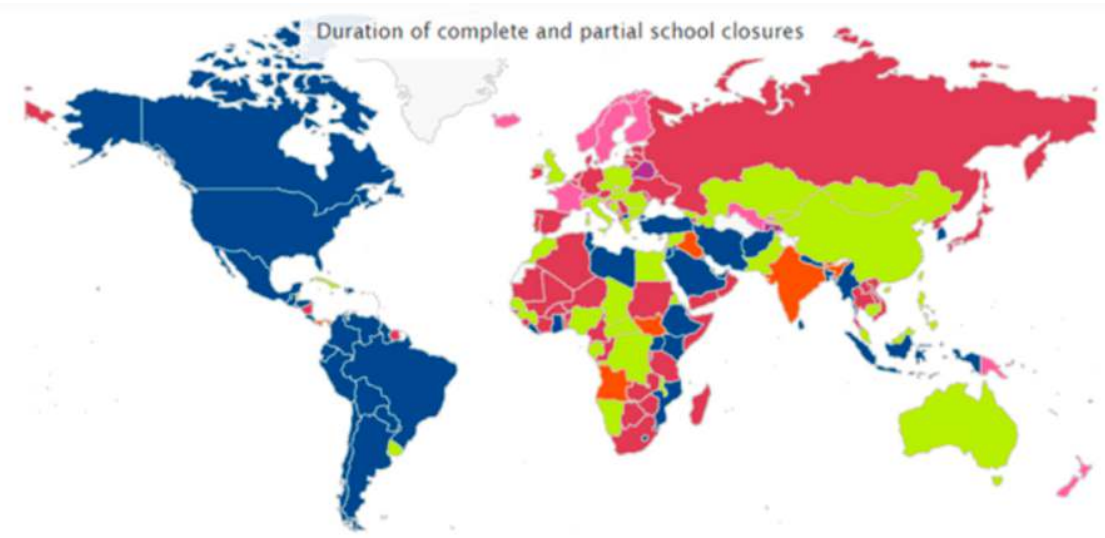

Figura 6. 
miento acceso y gobernanza, c) Participación e inclusión, d) Trabajo y seguridad económica (UNESCOb, 2020).

De igual manera y viendo lo complejo de las situaciones y marcos de trabajo en relación con la pandemia, en una reunión posterior, la UNESCO a través de las comisiones de estudio lanzó su compendio de ideas sobre una educación pospandemia, centrada en nueve pilares fundamentales (Figura 7).

En lo que ha considerado una apuesta complementaria que busca disminuir las brechas en materia de desigualdad, plantea nueve ideas para avanzar, sin olvidar lo básico del propósito de la educación, buscando reforzar los mejoramientos a través de los aprendizajes de los tiempos difíciles (UNESCOc, 2020).

\section{Banco Interamericano de Desarrollo (BID)}

El BID, en su informe llamado "El Futuro del Trabajo en América Latina (BID, 2019)", resalta dos elementos fundamentales para la región. Por una parte, el aumento significativo de la fuerza laboral en el

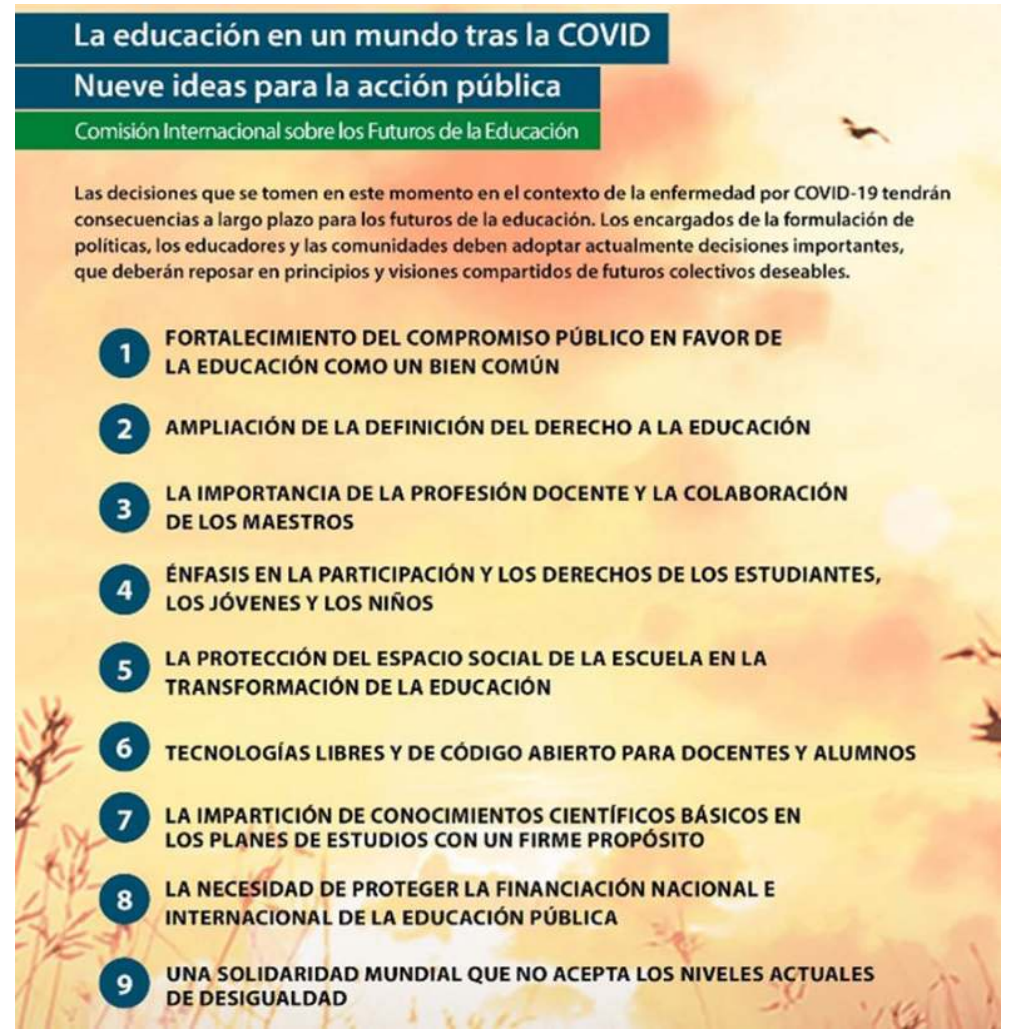

Figura 7. 
sector de la educación, en todos los niveles, comparado con otros. Esto sumado a las capacidades de los profesionales para definir el desenvolvimiento de la sociedad en Latinoamérica y el Caribe (BID, 2019) (Figura 8).

Dos aspectos clave para que el futuro de la región se mantenga apunta a que los profesionales del sector de la educación refuercen sus capacidades básicas, con relación al manejo de los seres humanos, así como al uso de las tecnologías de la información. Aunque el informe no describe los cambios fundamentales requeridos, sí enfatiza en las capacidades que los maestros deben desarrollar, para que el sistema esté acorde con las proyecciones (BID, 2019).

IESLAC (Instituto Internacional para la Educación Superior en América Latina y El Caribe)

En asocio con la UNESCO, surge el documento "COVID-19 y Educación Superior” (IESALC,2020), que plantea observaciones sobre cómo la educación superior debe enfrentar el momento de crisis generado por la pandemia; pero, sobre todo, las consideraciones para enfrentar el futuro, marcado por cambios profundos en el modelo de la educación superior. Destaca el esfuerzo en América Latina de cara a otras regiones del mundo y formula sus recomendaciones.

Ante los efectos de la pandemia en los seres humanos, sin precedentes, no es fácil pronosticar el futuro, sobre todo en temas de educación (IESLAC,2020).

Los efectos en todas las cadenas de la educación superior son notables, tanto en los estudiantes quienes han experimentado nuevas formas para recibir las clases, como en los profesores y profesionales de la educación, replanteando métodos de enseñanza y la forma de adaptarse a la situación, además de aprender sobre el uso de nuevas herramientas (Figura 9).

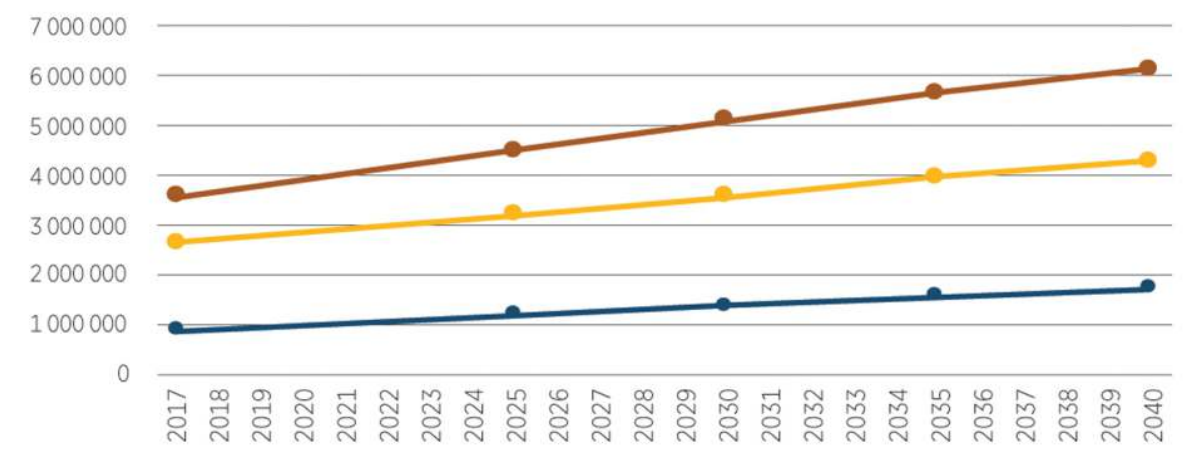

Figura 8. Proyecciones del número de docentes, por nivel 


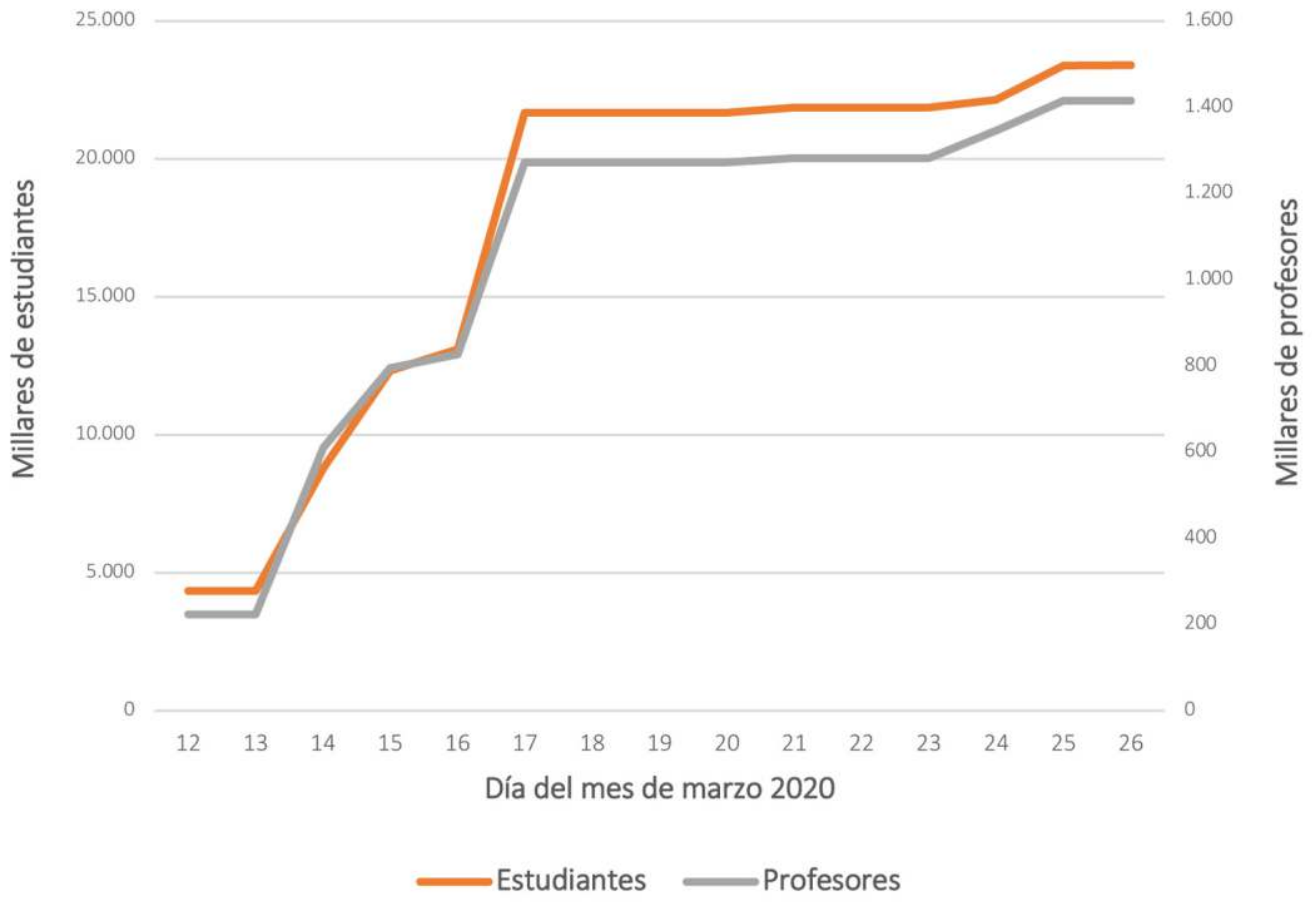

Figura 9.

Los impactos son evidentes en varios aspectos de corto y largo plazo. Los más relevantes se relacionan en el cuadro 1.

Dentro del informe se resaltan aspectos clave para enfrentar estos desafíos y los que vienen, centrados en la figura 11.

El mejor futuro de la educación girará en torno a repensar los modelos actuales de la misma (IESALC, 2020).

\section{Organización de Estados} Iberoamericanos para la Educación, la Ciencia y la Cultura (OEI)

En su reciente libro denominado "La Educación del Mañana: ¿Iner- cia o Transformación?" (OEI, 2020), la entidad muestra aspectos importantes sobre la forma cómo será la educación en los años venideros. Se pregunta si en el 2030 existirá la educación como la conocemos y explora diferentes escenarios.

Los autores de este extenso informe resaltan que la única certeza en la educación del futuro es la incertidumbre, concepto basado en los cambios actuales enfrentados por la humanidad.

Los factores clave que acompañarán la educación del futuro serán:

1. La revolución digital, como un elemento dinamizador de las so- 
Cuadro 1.

\begin{tabular}{|c|c|c|}
\hline Tipos & Corto plazo & Largo plazo \\
\hline Estudiantes & $\begin{array}{l}\text { Sin ideas claras sobre } \\
\text { cómo va a ser el proceso } \\
\text { de continuar. } \\
\text { Efectos en la vida } \\
\text { cotidiana y equilibrio } \\
\text { emocional. } \\
\text { Conectividad e inclusión. }\end{array}$ & $\begin{array}{l}\text { Costos y cargas } \\
\text { financieras. } \\
\text { Formas de aprendizaje y } \\
\text { ambientes híbridos del } \\
\text { futuro. } \\
\text { Movilidad y globalización. }\end{array}$ \\
\hline $\begin{array}{l}\text { Profesores y } \\
\text { profesionales de la } \\
\text { educación }\end{array}$ & $\begin{array}{l}\text { Disminución de la planta } \\
\text { por temporalidad. } \\
\text { Adaptabilidad a la } \\
\text { virtualidad permanente. } \\
\text { Inclusión de la tecnología } \\
\text { como un deber en el } \\
\text { marco pedagógico de la } \\
\text { formación. } \\
\text { Nuevo desarrollo de } \\
\text { cuerpo de habilidades y } \\
\text { capacidades para } \\
\text { impartir enseñanza }\end{array}$ & $\begin{array}{l}\text { Adaptación a nuevos } \\
\text { modelos de enseñanza. } \\
\text { Desarrollos de habilidad } \\
\text { por modelo de práctica } \\
\text { en ambientes híbridos. } \\
\text { Desafíos de la } \\
\text { conectividad en lugares } \\
\text { remotos. }\end{array}$ \\
\hline
\end{tabular}

Fuente: Elaboración propia.

ciedades, que con la presencia de la pandemia se convirtió en un acelerador de elevada importancia para la educación. Entre los factores clave señala la conectividad, la vida basada en algoritmos y la inteligencia artificial como piezas fundamentales del futuro.

2. Factores demográficos basados en la globalización, las migraciones, el envejecimiento de las poblaciones y el orden económi$\mathrm{co}$, hechos que han generado presiones importantes en los países, en términos de educación.
3. Las relaciones clave entre productividad y educación contempladas en el informe del (FEM, 2020).

4. La revolución del conocimiento entre generalidad y especificidad, alrededor de unos programas rígidos y con currículos específicos, a unos programas más adaptables y con currículos moldeables a las expectativas de las partes interesadas, que influyen en la productividad de los países.

En la declaración de la OECD, se plantea la necesidad de repensar la 

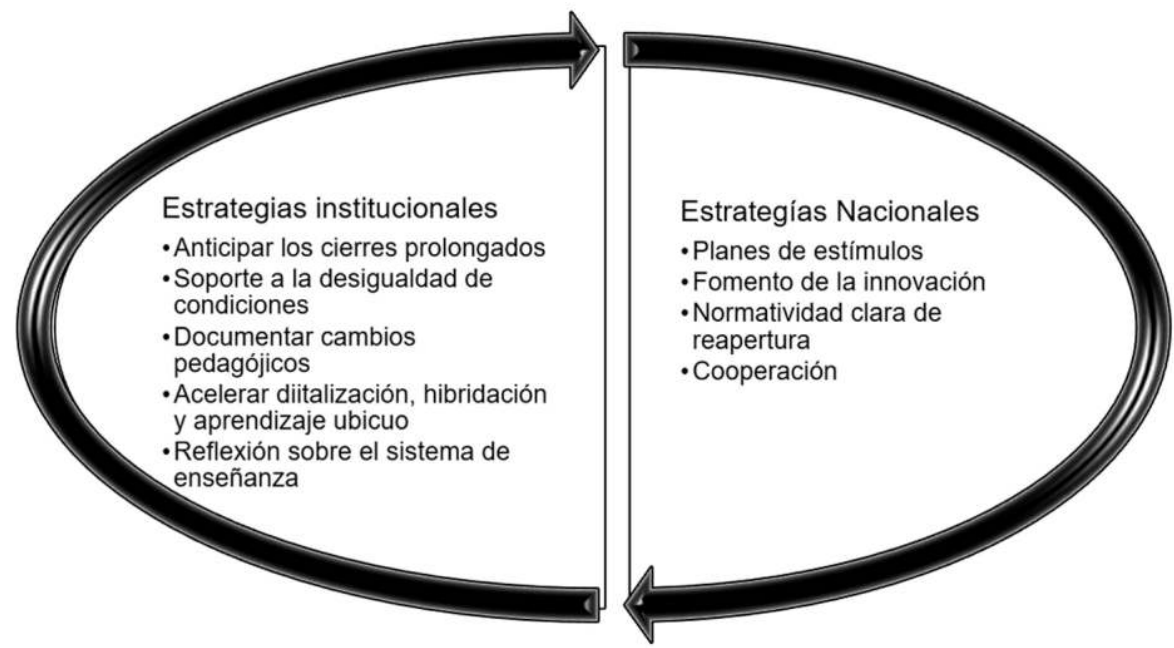

Fuente: Elaboración propia.

educación desde sus cimientos (OEI, 2020):

1. El futuro de la educación se escribe en el momento presente y con la velocidad que se requiere.

2. La innovación, el conocimiento y el crecimiento son elementos clave en los nuevos modelos educativos, en los que el conocimiento dejo de ser lineal y lento, la escuela el único lugar posible para adquirirlo, el profesor y los textos no son las únicas fuentes de información veraces y la rigidez de los programas ha dejado de ser funcional.

3. El sentido de educar cada vez más debe responder a la pregunta de ¿para qué? Ayudará a repensar si estamos preparados para afrontar los desafíos de un futuro de la educación.

4. Valoración del aparato educativo por parte de las personas, y so- ciedades como parte de mejoramiento del proceso.

5. Mejoramiento de la educación en todas sus etapas y la conexión sistémica del proceso.

6. Definitivamente la conectividad y la autonomía del proceso en las etapas más avanzadas de la educación serán piezas fundamentales.

7. En el arte de aprender se refuerza la idea de que las personas deben considerar el aprendizaje como un proceso paralelo a la vida, que no se detiene al terminar un ciclo, sino que debe permanecer durante la existencia para tener mayores oportunidades dentro del sistema.

8. Aprender a aprender, se convierte en una función primaria del aprendizaje desde las etapas más tempranas de la vida; no se fomenta como propósito culminar ciclos, sino la necesi- 
dad de ser aprendices permanentes. En este punto prima lo aprendido, la innovación, la flexibilidad y la diversidad, como elementos esenciales.

Según el informe de la OEI hay cuatro claves esenciales (Figura 12).

Se promueven las experiencias positivas de aprendizajes, la educación de las personas como un proceso de evolución y acompañamiento en el ciclo de la vida, el enfoque del bienestar emocional en el proceso de aprender, y un desarrollo cognitivo que le permita a los estudiantes interactuar y participar, cada vez con mayores criterios, habilidades y capacidades en unas sociedades más amplias y globales.

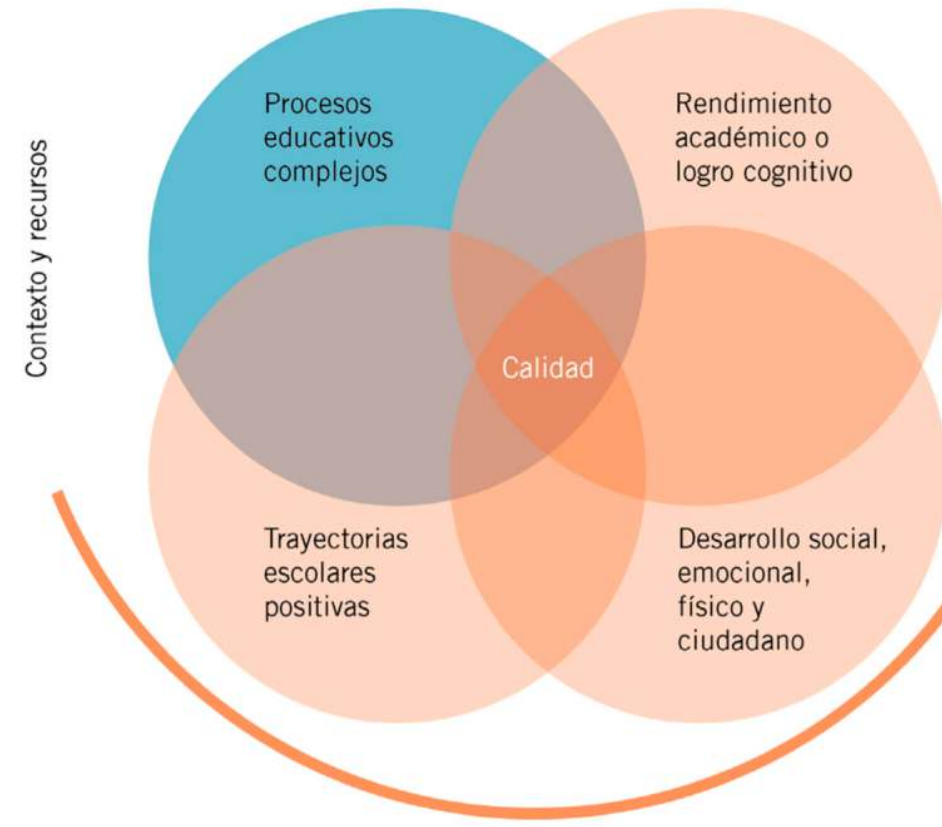

OCDE

La Organización para la Cooperación y el Desarrollo Económico (OCDE) lanzó en el año 2015 el proyecto denominado "El Futuro de la Educación y las Habilidades 2030 ", planteando como objetivo el trabajo colaborativo y el esfuerzo conjunto, para dar respuesta a temas fundamentales, como los conocimientos, habilidades, actitudes y valores, considerando los estudiantes del futuro y los requisitos para lograrlo (OECD, 2018).

Proyecto estructurado en varias fases con tales temáticas como ejes centrales.

Entre los principales ideales se observan: entender que las personas son una fuente de crecimiento integral; que su proceso de aprendi-
Resultados

Figura 12. 


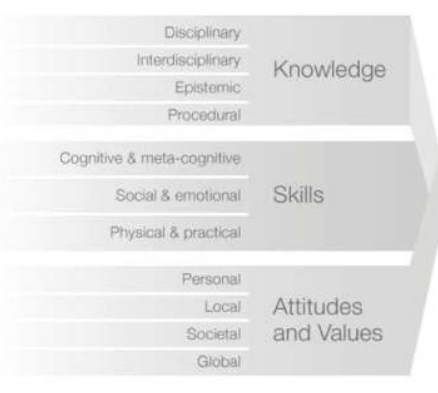

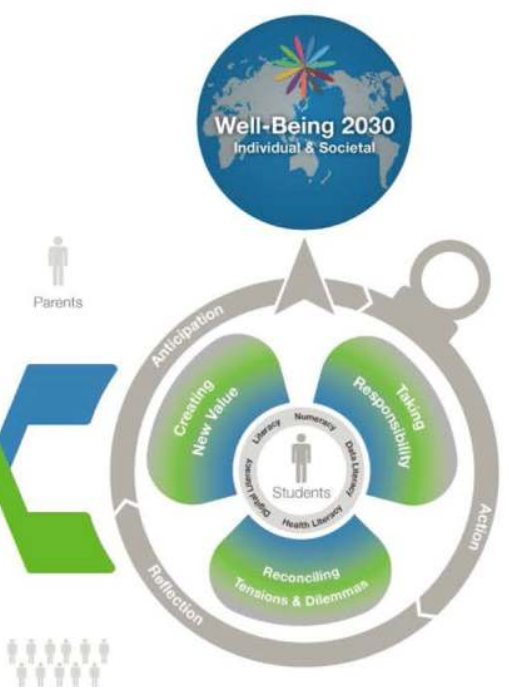

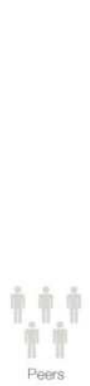

Figura 13.

zaje repercute en su bienestar y no se trata de una rueda suelta. De ahí que promulguen un marco de trabajo con una visión sistémica para el desarrollo de las futuras generaciones (Figura 13).

Así mismo la OECD, sabiendo que esto es un proyecto de largo aliento, ha venido trabajando en otros frentes muy importantes después del lanzamiento de su iniciativa, relacionados con los currículos, basados en cuatro asuntos importantes: 1 . El retraso en los cambios del currículo, 2. La demora en la toma de decisiones para cambiar los currículos, 3. Retraso en la implementación y 4 . El lapso de impacto entre las decisiones y los resultados mostrados al aplicar los currículos (OECD,2019).

En esa misma línea, la UNESCO para el año 2020 hace un aporte complementario pensando en la pandemia, en el que plantea escenarios posibles para la educación del futuro, en el marco de la incertidumbre y los escenarios posibles (OECD,2020) (Figura 14).

Este interesante ejercicio planteado busca alternativas, probar y experimentar posibles caminos y plantear visiones compartidas del futuro para apoyar el presente por el cual se está transitando (Figura 15).

El primer escenario es la escuela extendida, en la que se plantea la colaboración a nivel global, soportada en una fuerza de la tecnología y una individualización del aprendizaje; las estructuras y el proceso de mantienen igual. El segundo escenario denominado la educación tercerizada, supone la terminación total del modelo actual, el aprendi- 


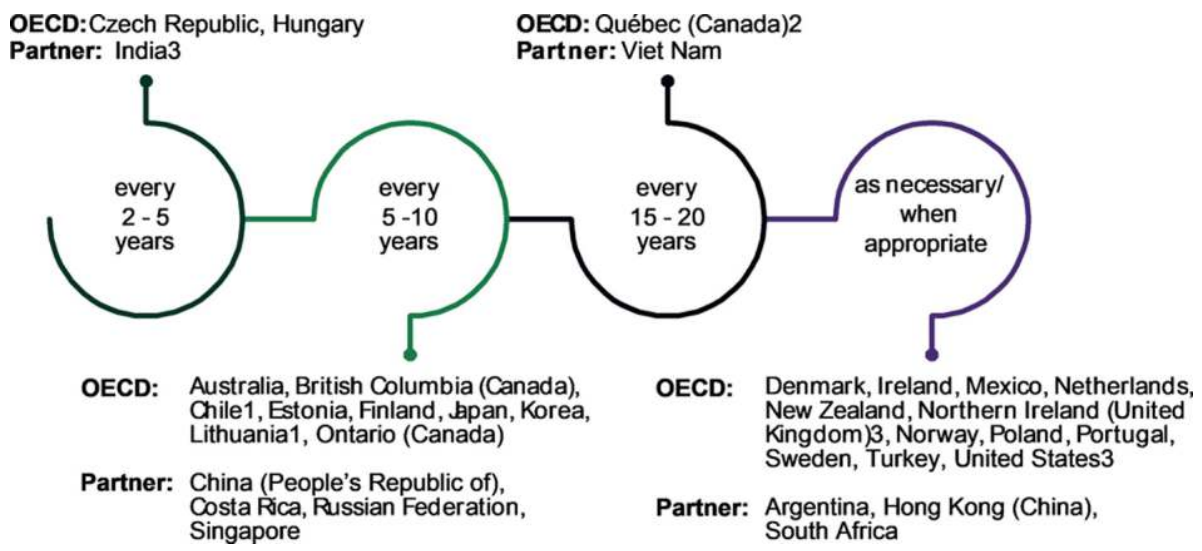

Figura 14. Ilustración Fuente: (OECDb,2019)

zaje se da en modelos diversos sin estar sujetos a estructuras, con privacidad y arreglos más individuales y la tecnología como un driver fundamental. El tercer escenario define a las escuelas como centros de aprendizajes, en los cuales el modelo tradicional permanece, pero la experimentación, y la diversidad de técnicas son la fuente natural del modelo educativo; las escuelas están más conectadas no solo en forma digital, sino en los contextos comunitarios los cuales pueden dar lugar a la innovación y el desarrollo social. El último escenario con relación a aprender sobre la marcha, en cualquier lugar y momento, elimina la diferencia entre educación formal e informal, entre las aulas

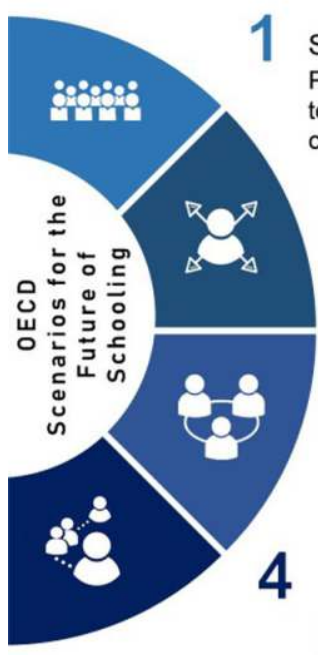

SCHOOLING EXTENDED

Participation in formal education continues to expand. International collaboration and technological advances support more individualised learning. The structures and processes of schooling remain.

\section{2 education outsourced}

Traditional schooling systems break down as society becomes more directly involved in educating its citizens. Learning takes place through more diverse, privatised and flexible arrangements, with digital technology a key driver.

\section{SCHOOLS AS LEARNING HUBS}

Schools remain, but diversity and experimentation have become the norm. Opening the "school walls" connects schools to their communities, favouring everchanging forms of learning, civic engagement and social innovation.

LEARN-AS-YOU-GO

Education takes place everywhere, anytime. Distinctions between formal and informal learning are no longer valid as society turns itself entirely to the power of the machine.

Figura 15. Ilustración Fuente: (OECD,2020) 
como centros y los hogares. Claramente la dependencia de la tecnología es una fuente fundamental, no solo como herramienta, sino como pieza clave del aprendizaje.

\section{Conclusiones}

La educación del futuro es un mundo de inciertos, muy interesantes de analizar y muy importantes de visualizar. Luego de revisadas algunas de las visiones más importantes de los entes multilaterales que han plasmado ideas, sobre cómo debe ser la educación del futuro y sus posibles escenarios y efectos futuros, la labor de trabajar por repensar y modelar la educación del mañana empieza definitivamente hoy.

A continuación, se plantea una serie de ideas centradas en visualizar algunos puntos comunes en los planteamientos revisados y en las visiones exploradas. No son una guía definitiva, sino son puntos de encuentro comunes para promover ideas hacia la educación del futuro.

1. La educación es una fuente inagotable de conexión entre la sociedad, la comunidad, la vida laboral, la economía y el desarrollo de la humanidad. Por eso es necesario que la educación desde ahora vaya pensando en proporcionar las herramientas para poder navegar en un futuro cada vez más incierto.

2. Todos apuntan a considerar el Objetivo de Desarrollo Susten- table (OBS), como acuerdo marco, lo que significa ir más allá de los detalles y los asuntos clave.

3. La tecnología es una pieza fundamental que requiere miradas completas encaminadas a eliminar las brechas en términos de disponibilidad para las distintas comunidades en el mundo, de manera que la transformación digital en la educación se produzca con rapidez. En términos generales, no se trata solamente de aspectos como disponer de mayores componentes tecnológicos, de un currículo, de la entrega del aprendizaje, del soporte al estudiante y la investigación, también es necesario revisar la cadena que implica la operación de las instituciones, las personas, además de la suma de muchas de las capacidades adicionales que sostienen al modelo educativo. (KPMG, 2020).

4. Es clara para todos la necesidad de revisar variables basadas en las disrupciones recientes, en relación con los nuevos modelos abruptamente impuestos por la pandemia y revisar en ellos, la actualización de los marcos de enseñanza, soportados no solo por la tecnología, sino por las nuevas formas de pedagogía dentro de las dinámicas propias del proceso de actualización.

5. Si bien cada país y Estados tienen sus propias dinámicas para 
que sus procesos de revisión de los sistemas educativos se den, las distintas visiones coinciden en que se trata de una tarea pendiente por realizar (Fiszbein. A., Stanton. S., 2018).

6. Hay consenso en que los cambios y las transformaciones no solo se den dar en los procesos de la educación superior, sino en todos los niveles de los procesos formativos, y en que deben ser analizados de manera sistémica pensando en que los impactos y los cambios.

7. Todos los enfoques contemplan aspectos tales como: El acceso a la educación; el conocimiento como pieza clave del desarrollo de las sociedades; la tecnología como pieza angular, debe contemplar el sentido de educar para la vida, capacidades y habilidades, como fundamento del desarrollo de las personas; las instituciones deben ser cada vez más interdisciplinares y transdisciplinares, pero también interinstitucionales, en las que se fomente la cooperación, colaboración y la cocreación, así como una fuerte investigación para ayudar a avanzar a la humanidad, en términos de equidad, calidad e inclusión, como desafíos importantes que deben ser tenidos en cuenta a corto, mediano y largo plazo. Es necesario conectar la vida, el desarrollo y la enseñanza, en especial en los niveles superiores, con base en las necesidades de la industria y el mercado, para dar lugar a profesionales capaces de resolver problemas con un pensamiento crítico para navegar en medio de la complejidad (GUNi,2020).

8. Todos coinciden en que no hay un solo futuro para la educación (Rieckmann. M, 2010).

9. Es prioritario la revisión de los currículos en el marco de experiencias compartidas para el beneficio común y el desarrollo de estrategias dentro del contexto global (Orr, D. et all. , 2020).

10. Se requieren tecnologías emergentes, flexibilidad del sistema, sociedad e investigación y acciones en el presente que impacten el futuro, como estrategias clave (Granizo. X., et all. 2020).

\section{Referencias}

Anderson. C. (2014). La Educación del Futuro y el Futuro de la Educación. Ceplan.

https://www.ceplan.gob.pe/wpcontent/uploads/files/Documentos/ foro_educacion_final_02-05-2014. pdf

Banco Interamericano de Desarrollo (BID) 2019 El futuro del trabajo en América Latina y el Caribe. https://publications.iadb.org/public ations/spanish/document/Educaci $\%$ C3\%B3n_y_salud_los_sectores _del_futuro_versi\% $\overline{\mathrm{C}} 3 \% \overline{\mathrm{B}} 3 \mathrm{n} \_$para _imprimir.pdf 
Bast G. (2019) The Future of Education and Labor. In: Bast G., Carayannis E., Campbell D. (eds) The Future of Education and Labor. Arts, Research, Innovation and Society. Springer, Cham.

https://doi.org/10.1007/978-3-03026068-2_2

Castaño, F. (2020) El futuro de la educación desafíos de lo presencial a lo virtual. Ed Universidad Santiago de Cali. Recuperado de:

https://repository.usc.edu.co/bitstre am/handle/20.500.12421/4694/El $\% 20$ futuro\%20de\%20la\%20educa ci\%C3\%B3n.pdf?sequence=1\&isA llowed=y

Fiszbein. A., Stanton. S. (2018). The future of education in Latin America and the Caribbean.

https://www.observatorioeducacion .org/sites/default/files/usaid-layout6.12.2018-final_pdf.pdf

Fore, F. (2021). Reimagine Education. WEF.

https://www.weforum.org/events/th e-davos-agenda-2021/sessions/re imagining-education-1

Fore. F. Gurg. A., Fortier. S. Goodwin. J.,2021) Reimagine Education. WEF.

https://www.weforum.org/events/th e-davos-agenda-2021/sessions/re imagining-education-1, https:// www.weforum.org/events/the-da vos-agenda-2021/sessions/reima gining-education

Foro Económico Mundial (FEM). (2020). Schools of the Future. Defining New Models of Education for the Fourth Industrial Revolution http://www3.weforum.org/docs/WE
F_Schools_of_the_Future_Report _2019.pdf

Foro Económico Mundial (FEMb). (2020). The Future of Jobs Report. http://www3.weforum.org/docs/WE F_Future_of_Jobs_2020.pdf

Guterres. A. (2021). Construir hoy el futuro de la educación. UN. https://www.un.org/es/coronavirus/ articles/future-education-here

Global University Network For Innovation (GNUi) 2020 Higher Education in the World 7: Humanities and Higher Education: Synergies between Science, Technology and Humanities.

http://www.guninetwork.org/files/do wnload_full_report_heiw7.pdf

Granizo. X., Burbano. A., Delgado. C. 2020. Escenarios prospectivos de la educación superior del Ecuador al 2030. Revista Espacios. Vol. 41 (28) 2020-Art 13.

http://www.revistaespacios.com/a2 Ov41n28/a20v41n28p13.pdf

Instituto Internacional para la Educación Superior en América Latina y el Caribe (IESALC) (2020). COVID19 y educación superior: De los efectos inmediatos al día después. http://www.guninetwork.org/files/co vid-19-060420-es-2.pdf

KPMG (2020) The future of higher education in a disruptive world. https://assets.kpmg/content/dam/k pmg/xx/pdf/2020/10/future-of-hi gher-education.pdf

Martínez. P., Vialta. J. (2021). Education as a pillar of the 2030 Agenda. Evolution and perspectives in the 
context of the Covid-19 crisis. Revista Idees

https://revistaidees.cat/en/educatio n-as-a-pillar-of-the-2030-agenda/

Naciones Unidas (UN). (2020). La educación durante la COVID-19 y después de ella.

https://www.un.org/sites/un2.un.or g/files/policy_brief___education_ during_covid-19_and_beyond_spanish.pdf

Organización de las Naciones Unidas para la Educación la Ciencia y la Cultura (UNESCO) (2019). "Los futuros de la educación aprender a transformarse.

https://es.unesco.org/futuresofedu cation/sites/default/files/202010/UNESCO $\% 20$-\%20Futures $\%$ 20 of $\% 20$ Education $\% 20-\% 20 B$ ro chure\%20-\%20SP._1.pdf

Organización de las Naciones Unidas para la Educación la Ciencia y la Cultura (UNESCOb) (2020). Visión y marco de los futuros de la educación. https://unesdoc.unesco.org/ ark:/48223/pf0000373208_spa/PD F/373208spa.pdf.multi

Organización de las Naciones Unidas para la Educación la Ciencia y la Cultura (UNESCOc) (2020). La educación en un mundo tras la COVID: Nueve ideas para la acción pública.

https://unesdoc.unesco.org/ark:/48 223/pf0000373717_spa/PDF/3737 17spa.pdf.multi

Organización de las Naciones Unidas para la Educación la Ciencia y la Cultura (UNESCO) (2021). La UNESCO revela una pérdida aproximada de dos tercios de un año académico en todo el mundo debido a los cierres de la COVID-19.

https://es.unesco.org/news/unesco -revela-perdida-aproximada-dostercios-ano-academico-todo-mun do-debido-cierres-covid-19

Organización de los Estados Iberoamericanos (OEI) (2022). La Educación del Mañana: ¿Inercia o Transformación?

https://oei.int/publicaciones/la-edu cacion-del-manana-inercia-o-trans formacion

Organización para la Cooperación y el Desarrollo Económico (OCDE) (2018) The Future of Education and Skills 2030

https://www.oecd.org/education/20 30-project/contact/E2030_Position _Paper_(05.04.2018).pdf

Organización para la Cooperación y el Desarrollo Económico (OCDE) (2019) What Students Learn Matters: Towards a 21st Century Curriculum.

https://www.oecd-ilibrary.org/si tes/d86d4d9a-en/1/3/1/index.html ?itemld=/content/publication/d86d 4d9a-en\&_csp_ff3034e $35 \mathrm{a} 61198$ 7aedaec8131c $\overline{4}$ a66f1 \&itemI GO =o ecd\&itemContentType=book\#figur e-d1e515

Orr, Dominic \& Luebcke, Maren \& Schmidt, J. \& Ebner, Markus \& Wannemacher, Klaus \& Ebner, Martin \& Dohmen, Dieter. (2020). Higher Education Landscape 2030: A Trend Analysis Based on the AHEAD International Horizon Scanning.10.1007/978-3-030-44897-4.

Rieckmann. M. 2010. Future-oriented higher education: Which key com- 
petencies should be fostered through university teaching and learning?

https://www.sciencedirect.com/scie nce/article/abs/pii/S001632871100 2448?via\%3Dihub

Soler, E. (2020). El mundo en 2021: diez temas que marcarán la agenda internacional. CIDOB Notes Internationals. 243.

https://www.cidob.org/publicacione s/serie_de_publicacion/notes_inter nacionals_cidob/243/el_mundo_en 2021_diez_temas_que_marcaran _la_agenda_internacional

Andrés R. Almanza J., Ms.C, CISM. Chief Growth Officer en CISOS.CLUB, Investigador en Ciberseguridad SegInfo y Liderazgo. | Executive Certificate in Cybersecurity Leadership \& Strategy by FIU University | Certificado como ISO 27001 Lead Implementer and 27005 Lead Manager from PECB | CISM, ITILv3, LPI| Certificado como Coach Profesional Internacional, Master in Leadership and Organizational Development with Coaching, Executive Master's in Leadership Skills Developed in Harvard, \& Coach Profesional avalado por International Coach Federation| Profesional en Ingenieria de Sistemas | especialista en seguridad en redes y máster en seguridad de la información. Docente del programa de maestría de la Universidad Externado de Colombia y de la Universidad de las Américas en Ecuador. Creador de la Comunidad CISOS.CLUB, CISOS-COL y CISOS-LATAM (Linkedin) y miembro del comité editorial de la revista sistemas de ACIS. 